\title{
miR-124/MCP-1 signaling pathway modulates the protective effect of itraconazole on acute kidney injury in a mouse model of disseminated candidiasis
}

\author{
XIAO-YUE LI ${ }^{1}$, YU-QI ZHANG ${ }^{1}$, GANG XU ${ }^{2}$, SHAO-HONG $\mathrm{LI}^{3}$ and HENG LI ${ }^{4}$ \\ ${ }^{1}$ Department of Critical Care Medicine, The Second Affiliated Hospital of Guilin Medical University, Guilin, \\ Guanxi 541199; ${ }^{2}$ Department of Geriatrics, Guangzhou First People's Hospital, Guangzhou, Guangdong 510000; \\ Departments of ${ }^{3}$ Emergency and ${ }^{4}$ Cardiovascular Medicine, TungWah Affiliated Hospital of Sun Yat-sen University, \\ Dongguan, Guangdong 523220, P.R. China
}

Received July 13, 2017; Accepted January 12, 2018

DOI: $10.3892 / \mathrm{ijmm} .2018 .3564$

\begin{abstract}
Previous studies have indicated that monocyte chemoattractant protein-1 (MCP-1), also referred to as $\mathrm{C}-\mathrm{C}$ motif chemokine ligand 2 , has a significant role in the pathogenesis of sepsis, however, how microRNAs (miRs) contribute to this process remains to be fully elucidated. In the present study, using a mouse model of disseminated candidiasis, the renoprotective effect of itraconazole (ITR) and adenovirus-delivered miR-124 was investigated. The mice were treated with ITR $(50 \mathrm{mg} / \mathrm{kg})$ or transfected with miR-124 mimics via tail-vein injection 7 days prior to Candida albicans infection. The survival outcome was monitored following candidiasis-induced sepsis with ITR or miR-124 mimics treatment. The levels of pro-inflammatory cytokines, including tumor necrosis factor- $\alpha$ (TNF- $\alpha$ ), interleukin- $1 \beta$ (IL-1 $\beta)$ and IL-6, were determined using enzyme-linked immunosorbent assays. The mRNA and protein levels were assayed using reverse transcription-quantitative polymerase chain reaction and western blot analyses, respectively. The results showed that ITR and miR-124 mimics improved the survival outcome in candidiasis-induced septic mice. The findings also indicated a significant downregulation in the serum levels of TNF- $\alpha$, IL- $1 \beta$ and IL- 6 in the septic mice treated with ITR or miR-124 mimics. Of note, ITR treatment significantly increased the expression of miR-124 and decreased the levels of MCP-1 in
\end{abstract}

Correspondence to: Dr Xiao-Yue Li, Department of Critical Care Medicine, The Second Affiliated Hospital of Guilin Medical University, 212 Renmin Road, Guilin, Guanxi 541199, P.R. China E-mail: xy_lig11@163.com

Dr Heng Li, Department of Cardiovascular Medicine, TungWah Affiliated Hospital of Sun Yat-sen University, 1 Dongcheng Road, Dongguan, Guangdong 523220, P.R. China

E-mail: heng_tw@163.com

Key words: itraconazole, acute kidney injury, microRNA-124, monocyte chemoattractant protein-1, sepsis the kidneys of the septic mice. It was also shown that the overexpression of miR-124 reduced the expression of MCP-1 and attenuated candidiasis-induced acute kidney injury (AKI) in septic mice. Transfection with miR-124 mimics was equivalent to ITR in reducing the excessive inflammatory response and renal lesions in septic mice. These results provided evidence supporting the use of miR-124 mimics as a therapeutic approach for attenuating candidiasis-induced AKI.

\section{Introduction}

Sepsis is a serious pathogenic infection and can deteriorate in a generalized immune response to systemic infection, which is a leading contributor to mortality rates in intensive care units (1). Candidiasis, particularly Candida albicans (C. albicans), is one of the leading worldwide nosocomial infections occurring following the onset of sepsis, and the mortality rate attributable to candidemia is $\sim 40 \%$ despite the use of antifungal agents $(2,3)$. In order to improve the treatment of candidemia-induced multiple organ failure, it is important to first understand its pathogenesis. A mouse model of disseminated C. albicans infection has been used extensively to examine the pathological process, and the kidney is the primary target organ (4). There is increasing evidence suggesting that the activation of the pro-inflammatory mechanism is involved in candidiasis-induced acute kidney injury (AKI) $(5,6)$. Interleukin-1 $\alpha$ (IL-1 $\alpha)$, IL-1 $\beta$, IL-6, tumor necrosis factor- $\alpha$ (TNF- $\alpha$ ) and interferon- $\gamma$ (IFN- $\gamma$ ) have been shown previously to be important in the pathogenesis of candidemia-induced renal injury $(7,8)$. In addition, chemokines and their receptors are reportedly induced in a fatal mouse model of invasive candidiasis $(5,7)$. These findings suggest that reducing the excessive production of inflammatory cytokines during candidemia is beneficial for attenuating AKI.

Itraconazole (ITR) is an azole broad-spectrum antifungal agent, and is orally administered for $C$. parapsilosis, C. albicans and C. glabrata infections (9-11). Generally, the treatment of fungal infections with ITR may be due not only to its direct antifungal effect, but also due to the induction of an immunomodulatory effect on the host defense 
systems via the regulation of cytokine production (12). ITR has an anti-inflammatory effect in vivo and in vitro $(13,14)$. In human lymphoid cells, ITR suppresses the accumulation of TNF- $\alpha$ and IFN- $\gamma$ messenger RNAs (mRNAs) in the presence of polyhydroxyalkanoate (15). In addition, ITR inhibits the TNF- $\alpha$-induced expression of CXCL10 in oral fibroblasts (16). In a murine model of chronic pulmonary paracoccidioidomycosis, ITR improves tissue lesions and immunomodulation through the regulation of pro-inflammatory cytokine levels (17). However, there has been no report on the protective effect of ITR in mice with renal injury.

A class of small non-coding RNAs (18-25 nucleotides) known as microRNAs (miRs), which regulate the translation of target mRNAs by binding to 3'-untranslated regions (3'-UTRs), have emerged as multifunctional post-translational modulators correlated with several diseases $(18,19)$. The association between sepsis and differentially expressed miRs has become a focus of investigations (20). C. albicans infection or bacterial cell surface lipopolysaccharide (LPS) treatment can induce the upregulation of miRs, including miR-146, miR-155, miR-455 and miR-125a, in macrophages (21). In our previous study, it was demonstrated that miR-204 and miR-211 were downregulated in the kidneys of septic mice, however, intravenous injection of miR-204 and miR-211 reversed sepsis-induced renal injury (22). Therefore, the identification of novel therapeutic targets based on an improved understanding of the molecular pathogenesis is required.

In the present study, ITR was identified as an antifungal agent against candidiasis-induced AKI through the inhibition of monocyte chemoattractant protein-1 (MCP-1). However, information on the effect of post-translational modulators on the nephroprotection of ITR in septic mice remains limited. Utilizing online prediction algorithms in the present study, MCP-1 was identified as a direct target of miR-124. It was recognized that miR-124/MCP-1 was closely associated with candidiasis-induced AKI, which may be a valuable molecular target for investigating the pathogenesis of renal dysfunction.

\section{Materials and methods}

Cell culture. 293T cells (American Type Culture Collection, Manassas, VA, USA) were incubated in Dulbecco's modified Eagle's medium (DMEM; Thermo Fisher Scientific, Inc., Waltham, MA, USA) and supplemented with 10\% FBS, $100 \mu \mathrm{g} / \mathrm{ml}$ streptomycin and $100 \mathrm{IU} / \mathrm{ml}$ penicillin (all from Sigma-Aldrich; Merck Millipore, Darmstadt, Germany).

Animal treatment. The experiments were approved by the Ethics Committee of the Second Affiliated Hospital of Guilin Medical University (Guilin, China) and were performed in accordance with its guidelines. A total of 120 male 8-week-old C57BL/6J mice (body weight, 20 $\pm 2 \mathrm{~g}$ ) were obtained from Charles River Laboratories (Wilmington, MA, USA) and were allowed to acclimate to the environment for 1 week. The mice were provided with free access to food and tap water, and were individually caged under a controlled temperature $\left(23 \pm 2^{\circ} \mathrm{C}\right)$ and humidity $(60 \pm 5 \%)$ with an artificial 12-h light/dark cycle. The mice were randomly divided into six groups $(n=20$ in each group) as follows: NC group injected with normal saline; CAN group infected with $C$. albicans via the injection of $1.0 \times 10^{7}$ colony-forming units intraperitoneally; ITR group treated with ITR (50 mg/kg; Xi'an-Janssen Pharmaceutical, Ltd., Xi'an, China) by intragastric administration; CAN+ITR group infected with $C$. albicans and co-treated with ITR $(50 \mathrm{mg} / \mathrm{kg})$; miR-Con group infected with $C$. albicans with co-injection of scramble sequence (miR-Con); miR-124 group infected with $C$. albicans with co-injection of miR-124 mimics. The $C$. albicans strain SC5314 was obtained from a fungal suspension (American Type Culture Collection) as described elsewhere (23) and cultivated in YPD (1\% yeast extract, $1 \%$ peptone and $2 \%$ dextrose) broth with overnight shaking at $30^{\circ} \mathrm{C}$. The systemic fungal infection was performed as in our previously described method (22).

Hematoxylin and eosin $(H \& E)$ staining. Kidney tissues were collected 7 days following $C$. albicans injection via intraperitoneal injection of sodium pentobarbital $(2 \% ; 150 \mathrm{mg} / \mathrm{kg}$; Sigma-Aldrich; Merck Millipore), and were fixed with 4\% formalin at room temperature for $24 \mathrm{~h}$ and paraffin-embedded. The tissues were then cut into $\sim 5-\mu$ m-thick sections, which were stained with $\mathrm{H} \& \mathrm{E}$ at room temperature for 1-2 min and visualized under a microscope (Leica DM 2500; Leica Microsystems GmbH, Wetzlar, Germany). Renal injury was assessed using a previously described $0-4$ point scale (24) as follows: 0 , none; $1,<10 \% ; 2,10-25 \% ; 3,25-75 \%$; or $4,>75 \%$.

Enzyme-linked immunosorbent assay (ELISA). The levels of inflammatory cytokines, TNF- $\alpha$ (cat. no. E-EL-M0049c), IL-1 $\beta$ (cat. no. E-EL-M0037c) and IL-6 (cat. no. E-EL-M0044c) were measured using mouse ELISA kits (Elabscience Biotechnology Co., Ltd., Wuhan, China) with a SpectraMax M5 ELISA plate reader (Molecular Devices, LLC., Sunnyvale, CA, USA), according to the manufacturer's protocol.

Measurement of glomerular filtration rate (GFR). Serum cystatin $\mathrm{C}(\mathrm{CysC})$ and $\beta 2$-microglobulin $(\beta 2-\mathrm{MG})$ are freely filtered by the glomerular membrane, which makes blood levels good indicators of GFR function. In the present study, CysC (cat. no. CYS4004) and $\beta 2-M G$ (cat. no. RQ9114) were measured using a RANDOX enzymatic creatinine assay (Randox Laboratories Ltd., Antrim, UK). Blood urea nitrogen (BUN) was measured via an enzymatic kinetic method using a commercial kit (cat. no. C013-2; Nanjing Jiancheng Bioengineering Institute, Nanjing, China). Serum creatinine ( $\mathrm{SCr}$ ) levels were measured using an autoanalyzer according to the manufacturer's protocol (cat. no. C011-1; Nanjing Jiancheng Bioengineering Institute).

Recombinant adenoviruses. Recombinant adenoviruses for the expression of miR-124 or control scrambled short hairpin RNA (miR-Con) were generated using the BLOCK-iT adenoviral RNAi expression system (Invitrogen; Thermo Fisher Scientific, Inc.) according to the manufacturer's protocol. High-titer stocks of amplified recombinant adenoviruses were purified as described previously (25). The viruses were diluted in PBS and administered at a dose of $10^{7}$ plaque-forming units per well in 12 -well plates, $10^{9}$ plaque-forming units per were administered to mice via tail-vein injection every other day for 7 days. 
Table I. Primers were used for reverse transcription-quantitative polymerase chain reaction of miRs and mRNAs.

\begin{tabular}{|c|c|c|}
\hline Gene & Forward primer $\left(5^{\prime}-3^{\prime}\right)$ & Reverse primer $\left(5^{\prime}-3^{\prime}\right)$ \\
\hline $\operatorname{miR}-124$ & CGTGTTCACAGCGGACCTTG & TGGTGTCGTGGAGTCG \\
\hline $\operatorname{miR}-290-5 p$ & ACACTCCAGCTGGGTTTCACGGGGGTATCA & TGGTGTCGTGGAGTCG \\
\hline $\operatorname{miR}-292-5 p$ & ACACTCCAGCTGGGGTTTTCUCGGGGGUCA & TGGTGTCGTGGAGTCG \\
\hline U6 & CGCTTCGGCAGCACATATACTAA & TATGGAACGCTTCACGAATTTGC \\
\hline MCP-1 & ATTTCCACACTTCTATGCCTCCT & ATCCAGTATGGTCCTGAAGATCA \\
\hline TNF- $\alpha$ & GCCACCACGCTCTTCTGTCTAC & GGGTCTGGGCCATAGAACTGAT \\
\hline IL-1 $\beta$ & ACCTTCCAGGATGAGGACATGA & CTAATGGGAACGTCACACACCA \\
\hline IL-6 & CACATGTTCTCTGGGAAATCG & TTGTATCTCTGGAAGTTTCAGATTG \\
\hline GAPDH & GCACCGTCAAGCTGAGAAC & TGGTGAAGACGCCAGTGGA \\
\hline
\end{tabular}

miR, microRNA; MCP-1, monocyte chemoattractant protein-1; TNF- $\alpha$, tumor necrosis factor- $\alpha$; IL, interleukin; GAPDH, glyceraldehyde 3-phosphate dehydrogenase.

Transfection with miR-124 mimics and miR-Con. The sequences of the miR-124 mimics (5'-UAAGGCACGCGG UGAAUGCC-3') and miR-Con (5'-UCCAAACAUCGGUGA AUGCC-3') were synthesized by Guangzhou RiboBio Co., Ltd. (Guangzhou, China). The 293T cells were transfected using Lipofectamine 2000 (Invitrogen; Thermo Fisher Scientific, Inc.) at a final concentration of $50 \mathrm{nM}$. At $48 \mathrm{~h}$ post-transfection, the cells were harvested for analysis.

Dual-luciferase reporter gene assay. The wild-type (WT, 5'-GACUCGGACUGUGUGCCUUA-3') and mutant-type (MUT, 5'-GACUCGGACUGUGUAUUCCA-3') 3'-UTR of MCP-1 were synthesized by PCR, and the fragments were subcloned into the pmirGLO Dual-Luciferase miRNA target expression vector (Promega Corp., Madison, WI, USA). The potential binding site between miR-124 and MCP-1 was obtained using online prediction software (miRanda-mirSVR; http://www.microrna.org), miRDB (http://www.mirdb. org/miRDB/) and TargetScan (http://www.targetscan.org/). The 293T cells were transfected with luciferase reporter vectors containing the WT and MUT MCP-1-3'-UTR (0.5 $\mu \mathrm{g})$. The miR-124 mimics or miR-Con were co-transfected at $50 \mathrm{nM}$. The luciferase activity was measured using the Dual Luciferase Reporter ${ }^{\circledR}$ assay system (cat. no. E1960; Promega Corp.) on a Luminoskan $^{\text {TM }}$ Ascent Microplate Luminometer (Thermo Fisher Scientific, Inc.). The dual-luciferase reporter gene assay was performed as previously described (22).

RNA analysis and RT-qPCR analysis. Total RNA was extracted using TRIzol (Invitrogen; Thermo Fisher Scientific, Inc.) according to the manufacturer's protocol. The cDNA was synthesized by RT reactions with $2 \mu \mathrm{g}$ of total RNA using moloney murine leukemia virus reverse transcriptase (Invitrogen; Thermo Fisher Scientific, Inc.) according to the manufacturer's protocol. The mRNA and miR levels were determined using the TaqMan RT-qPCR ELISA (Thermo Fisher Scientific, Inc.) on an ABI 7500 Real-Time PCR system (Applied Biosystems; Thermo Fisher Scientific, Inc.). A total of $20 \mu \mathrm{l}$ reaction mixture includes cDNA $(100 \mathrm{ng})+$ DEPC $\mathrm{H}_{2} \mathrm{O}(9 \mu \mathrm{l}), 300 \mathrm{nM}$ of each primer, $2 \mathrm{X}$ buffer $(10 \mu \mathrm{l})$ and $20 \mathrm{X}$ TaqMan Gene Expression Assay $(1 \mu \mathrm{l})$. The thermocycling reactions were as follows: $95^{\circ} \mathrm{C}$ for $10 \mathrm{~min}$, followed by 40 cycles of $95^{\circ} \mathrm{C}$ for $15 \mathrm{sec}, 60^{\circ} \mathrm{C}$ for $30 \mathrm{sec}$, and $72^{\circ} \mathrm{C}$ for $30 \mathrm{sec}$. The relative expression levels of $\mathrm{miR}$ and $\mathrm{mRNA}$ were calculated using the $2^{-\Delta \Delta \mathrm{Cq}}$ method (26) and normalized to the internal control U6 and glyceraldehyde 3-phosphate dehydrogenase, respectively. The primers were synthesized by Sangon Biotech Co., Ltd. (Shanghai, China) as shown in Table I.

Western blot analysis. Protein was extracted using Radioimmunoprecipitation Assay Lysis Buffer (Beyotime Institute of Biotechnology, Haimen, China). The concentration was determined using the Bicinchoninic Acid Kit for Protein Determination (Sigma-Aldrich; Merck KGaA). Samples containing $50 \mu \mathrm{g}$ of protein were separated using 10\% SDS-PAGE gel and transferred to nitrocellulose membranes (Bio-Rad Laboratories, Inc., Hercules, CA, USA). Following blocking with 5\% (w/v) non-fat dry milk in TBS and $0.1 \%(\mathrm{w} / \mathrm{v})$ Tween-20 (TBST), Primary antibodies MCP-1 (cat. no. sc-1784; dilution 1:500) and $\beta$-actin (cat. no. sc-130065; dilution 1:2,000) were obtained from Santa Cruz Biotechnology, Inc. (Santa Cruz, CA, USA) and incubated for $2 \mathrm{~h}$ at room temperature. The appropriate horseradish peroxidase-conjugated secondary antibody (cat. no. sc-516102; dilution 1:10,000) was purchased from Santa Cruz Biotechnology, Inc. and incubated for $1 \mathrm{~h}$ at room temperature. Western blot analysis was performed as previously described (22). Protein bands were densitometrically assessed using Quantity One ${ }^{\circledR}$ software (version 4.5; Bio-Rad Laboratories, Inc.).

Statistical analysis. Data are presented as the mean \pm standard deviation for each group. All statistical analyses were performed using PRISM version 5.0 (GraphPad Software, Inc., La Jolla, CA, USA). Inter-group differences were analyzed using one-way analysis of variance, followed by Tukey's post-hoc test for multiple comparisons. The log-rank test was used to compare group survival trends. $\mathrm{P}<0.05$ was considered to indicate a statistically significant difference.

\section{Results}

ITR improves survival outcome following candidiasis. C. albicans injection induced animal death within $24 \mathrm{~h}$, with 


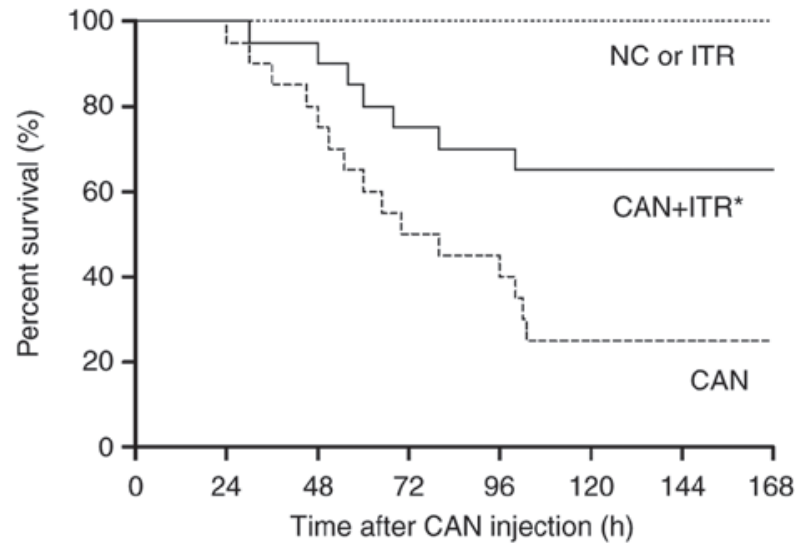

Figure 1. ITR improves survival outcome following candidiasis. C57BL/6 J mice were injected with $C$. albicans $\left(1.0 \times 10^{7}\right.$ colony-forming units) intraperitoneally with or without ITR $(50 \mathrm{mg} / \mathrm{kg})$. Animal survival was recorded for the duration of the investigation. ${ }^{*} \mathrm{P}<0.05$, compared with the CAN group. ITR, itraconazole; CAN, Candida albicans.

$50 \%$ mortality by $70 \mathrm{~h}$ post-C. albicans injection, and $25 \%$ of animals surviving at day 7 . However, in the septic mice treated with ITR, no death occurred until $30 \mathrm{~h}$ post- $C$. albicans injection, with $65 \%$ of the ITR-treated mice surviving for the duration of the investigation (Fig. 1).

ITR inhibits systemic and local inflammatory responses in septic mice. MCP-1 is a member of the CC chemokine family and it is an important molecule for monocyte recruitment under acute inflammatory conditions, regulating the progression of inflammation through the production of pro-inflammatory cytokines (27). A study by Labbe et al showed that antibody neutralization of MCP-1 prevented the endotoxin-induced upregulation of IL-1 $\alpha$, IL-1 $\beta$ and IL-6 in the diaphragm (28). Therefore, the present study investigated the levels of MCP-1 in septic mice. The results showed that the mRNA (Fig. 2A) and protein (Fig. 2B) expression levels of MCP-1 in the kidneys from septic mice were significantly higher, compared with those in the control group, which were markedly decreased by ITR (Fig. 2A and B). The serum and renal levels of pro-inflammatory cytokines (TNF- $\alpha$, IL-1 $\beta$ and IL-6) were determined. As shown in Fig. $2 \mathrm{C}$ and D, compared with the control group, the serum and renal levels of pro-inflammatory cytokines were significantly increased in the septic mice. Treatment with ITR for 7 days resulted in a decrease of pro-inflammatory cytokines in septic mice, suggesting that ITR inhibited the excessive systemic and local inflammatory response in candidiasis-induced AKI mice.

ITR alleviates candidiasis-induced AKI. BUN is an indicator of kidney impairment, and SCr represents a good indicator of the severity of renal impairment, particularly of GFR (29). To evaluate renal damage, BUN and SCr were measured in healthy and septic mice with or without ITR treatment. As shown in Fig. 3A and B, candidiasis induced significant increases in BUN and SCr levels, which were significantly attenuated by treatment with ITR. In addition, the present study analyzed the GFR by measuring CysC and serum $\beta 2-\mathrm{MG}$ under different treatment conditions. As shown in Fig. 3C and D, CysC and $\beta 2-\mathrm{MG}$ levels were significantly higher in the septic mice, compared with those in the control group, however, ITR
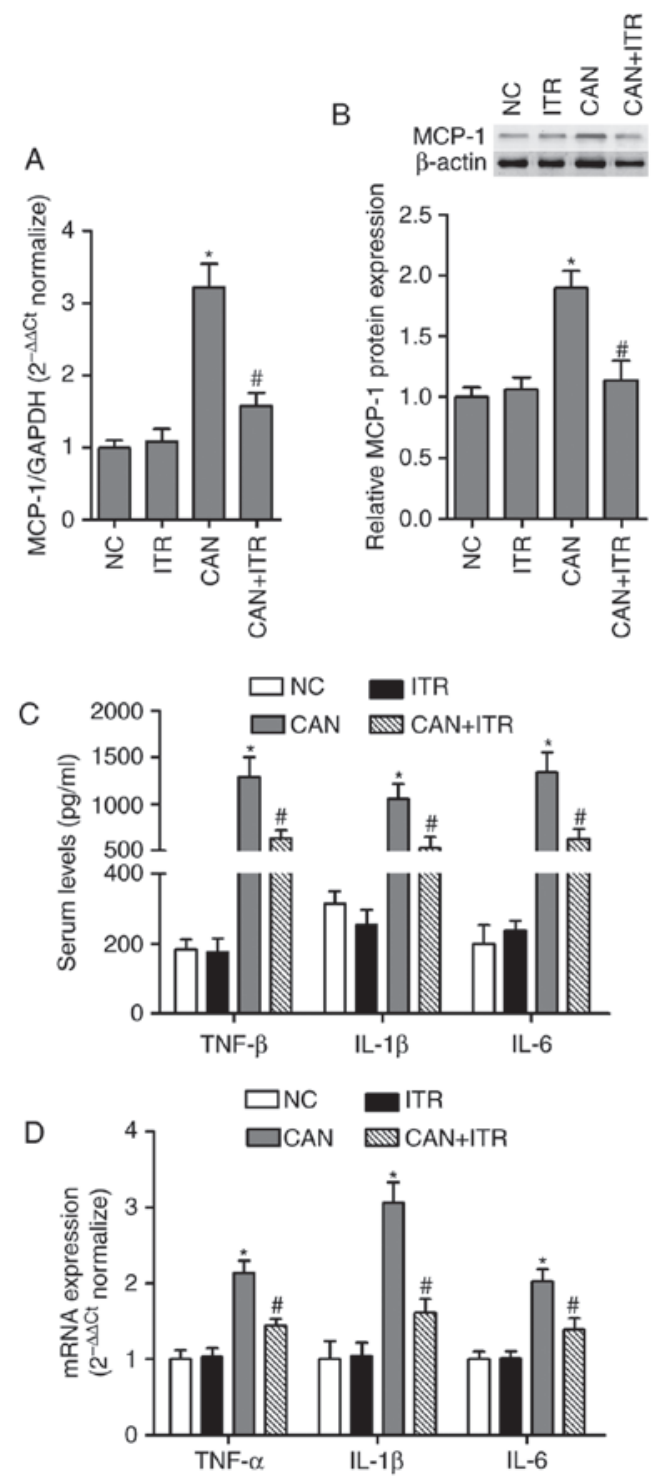

Figure 2. ITR inhibits systemic and local inflammatory responses in septic mice. mRNA and protein expression levels were analyzed by (A) RT-qPCR and (B) western blot analyses in the kidneys of septic mice with or without ITR treatment. (C) Levels of inflammatory cytokines, TNF- $\alpha$, IL- $1 \beta$ and IL-6, in serum were measured using mouse ELISA kits. (D) mRNA levels of TNF- $\alpha$, IL- $1 \beta$ and IL- 6 were analyzed by RT-qPCR analysis in the kidneys from septic mice with or without ITR treatment. " $\mathrm{P}<0.05$ compared with the NC group; ${ }^{\#} \mathrm{P}<0.05$ compared with the $\mathrm{CAN}$ group. TNF- $\alpha$, tumor necrosis factor- $\alpha$; IL, interleukin; ITR, itraconazole; CAN, Candida albicans; NC, negative control; RT-qPCR, reverse transcription-quantitative polymerase chain reaction.

treatment significantly reduced the increased levels of CysC and $\beta 2-\mathrm{MG}$ in the septic mice. In accordance with these data, improved renal pathological changes were observed in the ITR-treated septic mice. As shown in Fig. 4A and B, the induction of candidiasis produced focal and segmental hyperplasia with diffuse mesangial proliferation and glomerular lesions. By contrast, the damage was markedly attenuated in the ITR-treated group.

$I T R$ regulates the expression of $m i R-124$ in the kidneys of septic mice. Based on the experiments, MCP-1 was closely associated with candidiasis-induced AKI. To investigate whether MCP-1 may be regulated by miRs, online prediction software 

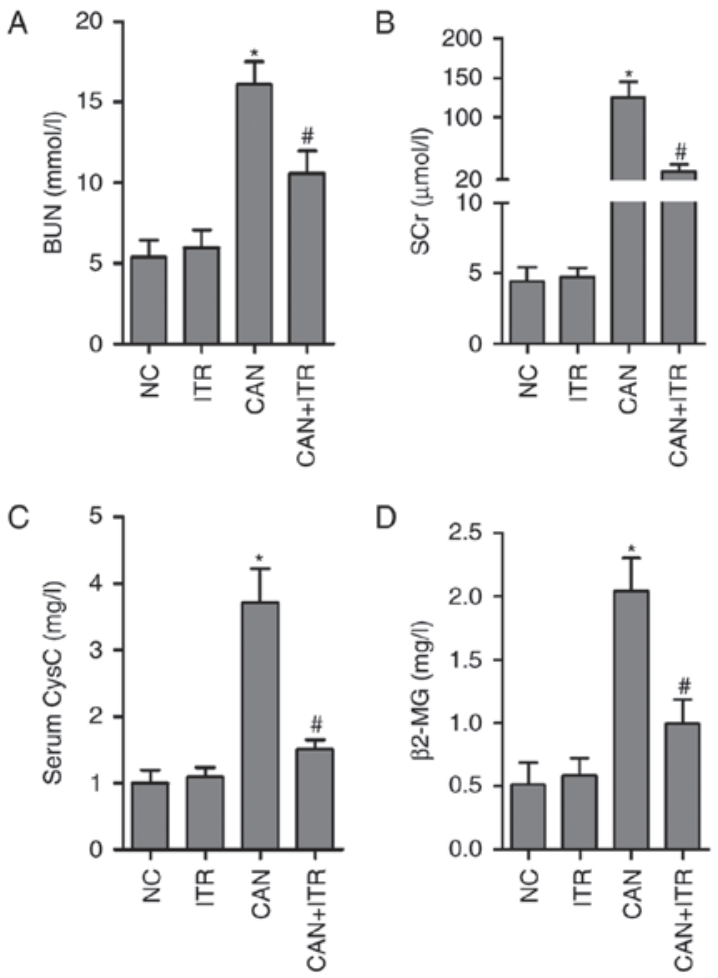

Figure 3. ITR alleviates candidiasis-induced acute kidney injury. The levels of (A) BUN, (B) SCr, (C) CysC and (D) $\beta 2-\mathrm{MG}$ were measured in healthy and septic mice with or without ITR treatment to evaluate renal injury. ${ }^{\text {* }} \mathrm{P}<0.05$ compared with the $\mathrm{NC}$ group; ${ }^{\prime} \mathrm{P}<0.05$ compared with the $\mathrm{CAN}$ group. $\mathrm{BUN}$, blood urea nitrogen; $\mathrm{SCr}$, serum creatinine; CysC, cystatin C; $\beta 2-\mathrm{MG}, \beta 2$-microglobulin; ITR, itraconazole; CAN, Candida albicans; NC, negative control.

(miRanda-mirSVR, miRDB and TargetScan) was used for prediction. It was found that miR-124 was overlapping in the miRanda-mirSVR and TargetScan databases, and miR-290-5p and miR-292-5p were overlapping in the miRanda-mirSVR and miRDB databases. Therefore, the expression levels of miR-124, miR-290-5p and miR-292-5p were measured in the kidneys from septic mice. The results demonstrated that the levels of miR-124 were significantly decreased in the kidneys from the C.albicans-injected mice, compared with those in the healthy mice (Fig. 5). However, there was no significant difference in miR-290-5p or miR-292-5p between the CAN group and NC group (Fig. 6A and B). Of note, ITR-treated reversed the candidiasis-induced downregulation of miR-124 in the kidneys of the septic mice (Fig. 5), suggesting that the overexpression of miR-124 contributed to the improved candidiasis-induced AKI. To investigate whether MCP-1 was a direct target of miR-124, the online prediction software was used for prediction. The results showed that the 3'-UTR of MCP-1 contained one conserved binding site of miR-124 (Fig. 7A). To confirm this, a luciferase activity assay was performed on the $293 \mathrm{~T}$ cells. As shown in Fig. 7B, transfection with miR-124 mimics significantly reduced the luciferase activity elicited by the luciferase vector carrying the complementary sequence with the WT 3'-UTR of MCP-1. Following transfection with miR-124 mimics, no significant difference was observed in luciferase enzyme activity between the $\mathrm{NC}$ group and the reporter vector group containing the MUT 3'-UTR of MCP-1. These findings demonstrated that MCP-1 was a direct target gene of miR-124 by binding with its 3'-UTR.
A

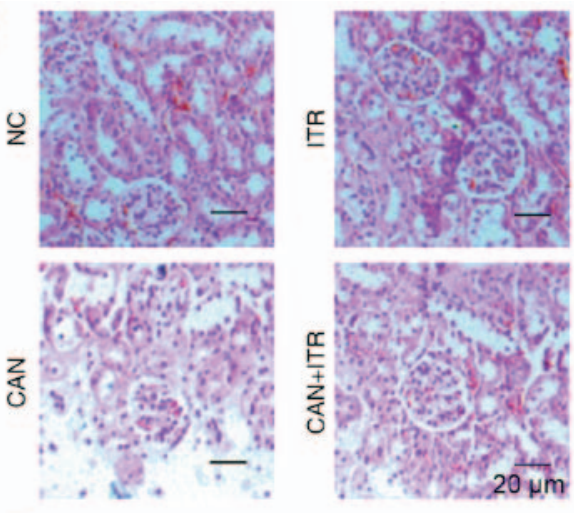

B

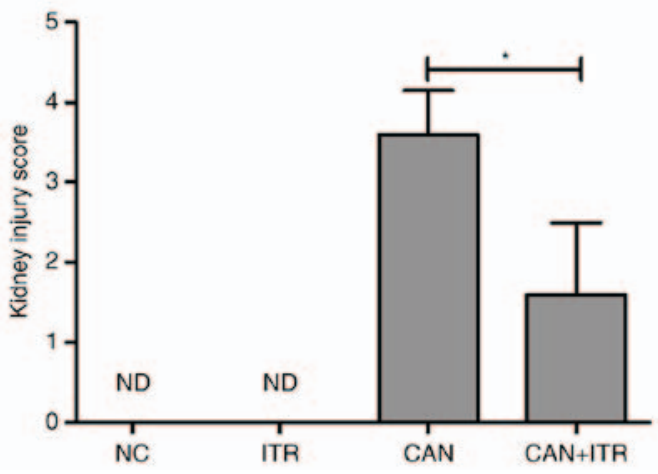

Figure 4. ITR alleviates candidiasis-induced acute kidney injury. (A) H\&E staining was performed in healthy and septic mice with or without ITR treatment to evaluate renal injury (magnification, x200). (B) Kidney injury scores are shown for each group. ${ }^{*} \mathrm{P}<0.05$ compared with the CAN group. ITR, itraconazole; CAN, Candida albicans; NC, negative control; ND, not detected.

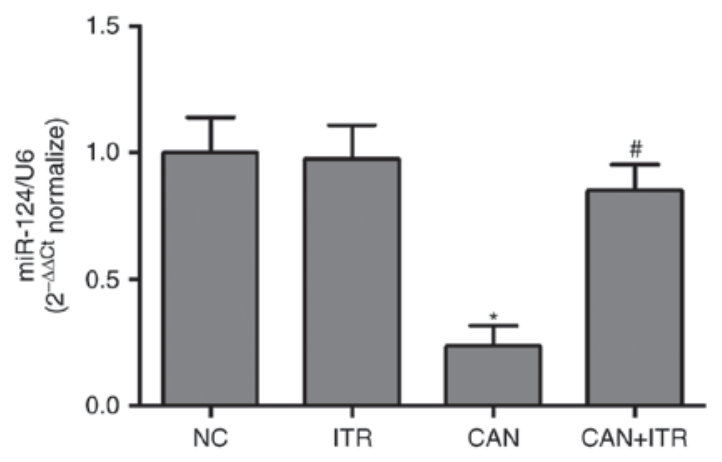

Figure 5. ITR regulates expression of miR-124 in the kidneys of septic mice. The levels of miR-124 were analyzed by reverse transcription-quantitative polymerase chain reaction analysis in the kidneys of septic mice with or without ITR treatment. ${ }^{*} \mathrm{P}<0.05$ compared with the $\mathrm{NC}$ group; ${ }^{*} \mathrm{P}<0.05$ compared with the CAN group. miR, microRNA; ITR, itraconazole; CAN, Candida albicans; NC, negative control.

Overexpression of miR-124 improves survival outcome following candidiasis. In septic mice, miR-Con transfection had no significant effect on survival outcome. However, the septic mice transfected with miR-124 mimics began to die at $35 \mathrm{~h}$, with $60 \%$ of the septic mice surviving for the duration of the investigation (Fig. 8).

Intravenous injection of miR-124 inhibits the expression of MCP-1, inflammatory cytokines and AKI in septic mice. To investigate the effects of miR-124 on the inflammatory response and sepsis-induced AKI in vivo, male C57BL/6J 

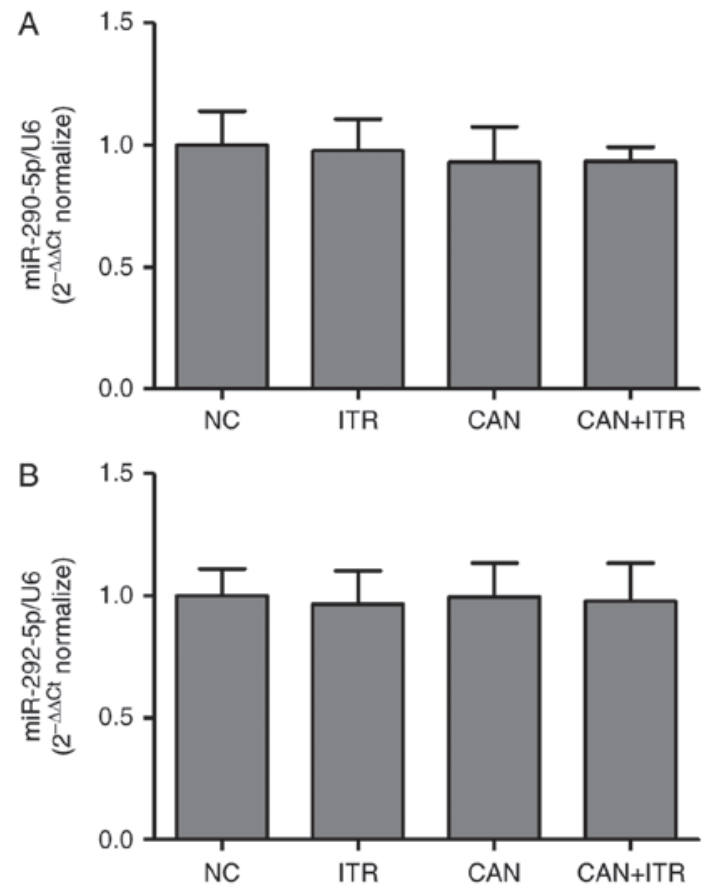

Figure 6. Expression of miR-290-5p and miR-292-5p. Levels of (A) miR-290-5p and (B) miR-292-5p were analyzed by reverse transcription-quantitative polymerase chain reaction analysis in the kidneys of septic mice with or without ITR treatment. miR, microRNA; ITR, itraconazole; CAN, Candida albicans; NC, negative control.
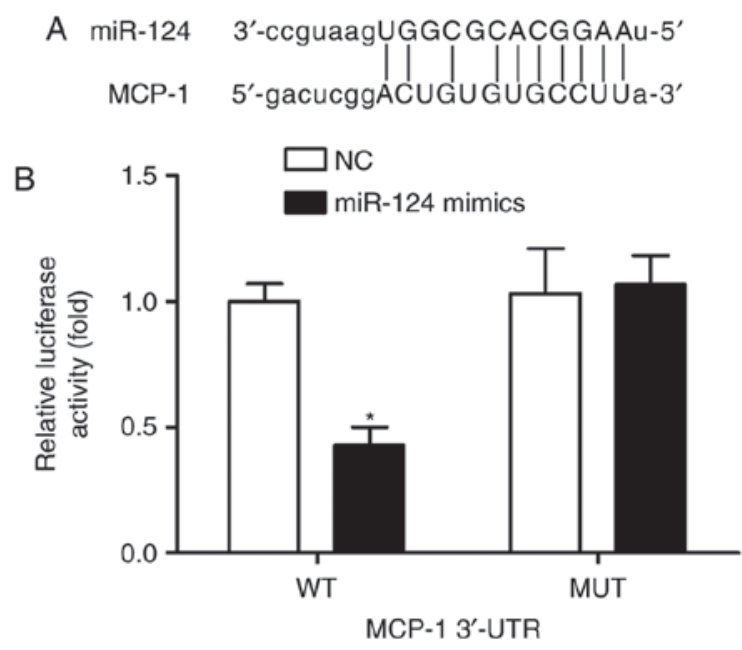

Figure 7. MCP-1 is a direct target gene of miR-124. (A) Schematic representation of the putative miR-124 binding site in the 3'-UTR of MCP-1 was predicted using online databases. (B) $293 \mathrm{~T}$ cells were co-transfected with the WT or MUT MCP-1-3'-UTR and miR-590-3p mimics or miR-Con, and a luciferase activity assay was performed. ${ }^{*} \mathrm{P}<0.05$ compared with the NC group ( $\mathrm{n}=3$ in each group). WT, wild-type; MUT, mutant; miR, microRNA; MCP-1, monocyte chemoattractant protein-1.

mice were infected with miR-124 mimics and miR-Con via tail-tail injection, and it was found that levels of miR-124 were significantly increased in the kidneys, compared with those in the NC group or miR-Con group (Fig. 9A). To determine the effects of miR-124 on inflammatory signaling, the effects of the overexpression of miR-124 on MCP-1 were examined in the kidneys of the mice. The mRNA and protein levels of MCP-1 were significantly inhibited in the kidneys of mice transfected

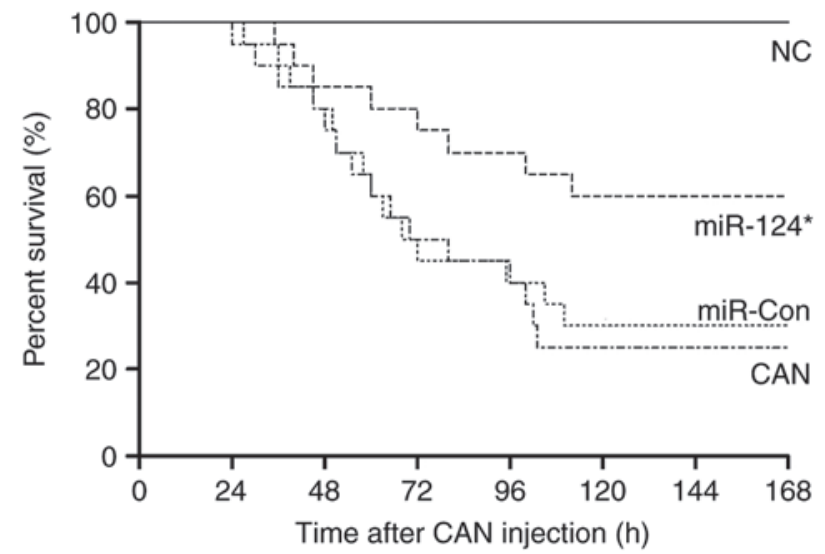

Figure 8. Overexpression of miR-124 improves survival outcome following candidiasis. C57BL/6J mice were injected with Candida albicans (1.0x $10^{7}$ colony-forming units) intraperitoneally with or without miR-124 mimics. Animal survival was recorded for the duration of the study. ${ }^{*} \mathrm{P}<0.05$ compared with the CAN group. miR, microRNA; CAN, Candida albicans; $\mathrm{NC}$, negative control.

with miR-124, compared with those in the miR-Con group (Fig. 9B-D). The effects of miR-124 mimics on the serum levels of inflammatory cytokines associated with MCP-1 were also examined in septic mice. It was found that the serum levels of TNF- $\alpha$, IL-1 $\beta$ and IL- 6 were markedly decreased by miR-124 mimics in septic mice (Fig. 9E). Furthermore, the miR-124 mimics led to significant decreases in the levels of BUN and SCr in serum of the septic mice (Fig. 9F and G), suggesting that miR-124 may improve the inflammatory response and $\mathrm{AKI}$ in septic mice through the inhibiting the expression of MCP-1.

\section{Discussion}

Experimental and clinical data support that candidiasis is a dominant factor for AKI $(22,30)$. There are no effective therapies in candidiasis-induced AKI. In order to improve clinical outcomes, understanding the exact molecular mechanisms in candidiasis-induced AKI is considered essential. The present study used a mouse model of disseminated candidiasis, and the findings indicated that the upregulation of MCP-1 levels and inhibition of miR-124 were involved in candidiasis-induced AKI. In addition, a bioinformatics prediction approach showed that miR-124 was targeted to MCP-1 by binding with its 3 '-UTR. The data revealed molecular insights into the involvement of miR-124 and its direct target MCP-1 in modulating sepsis-induced renal injury.

Previous studies have demonstrated that inflammation is pivotal in the pathogenesis of AKI, and that MCP-1, which is considered a key inducer of the proinflammatory response, has been shown to be linked with renal perfusion, glomerular filtration and glomerular lesions $(31,32)$. Munshi et al showed that the pathologic increase in the expression of MCP-1 originates from the diseased kidney, and MCP-1 offers potential as a biomarker of AKI (31). MCP-1 is known to contribute to the toxic effect of LPS in vivo and in vitro $(33,34)$; therefore, attenuating a pathologic increase in the expression of MCP-1 may be important in controlling the occurrence and development of AKI. The present study 
A

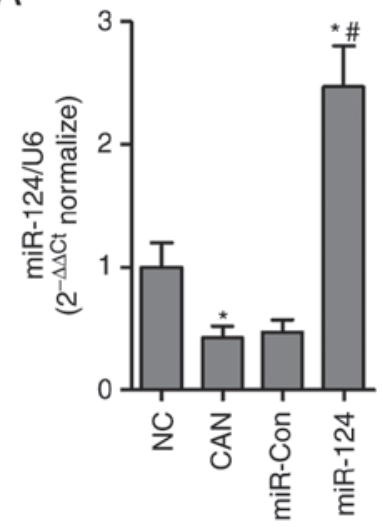

B

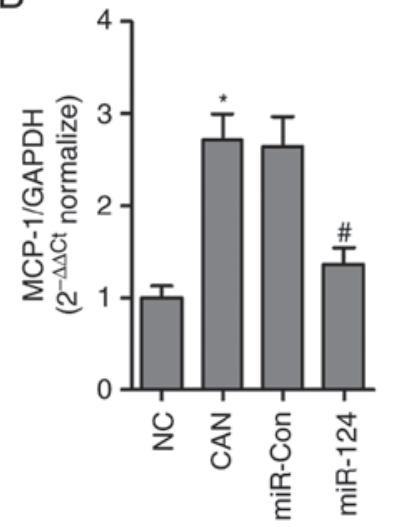

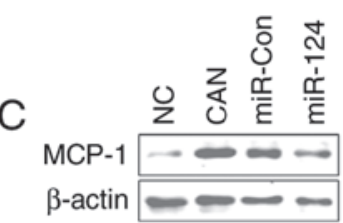

$\mathrm{F}$

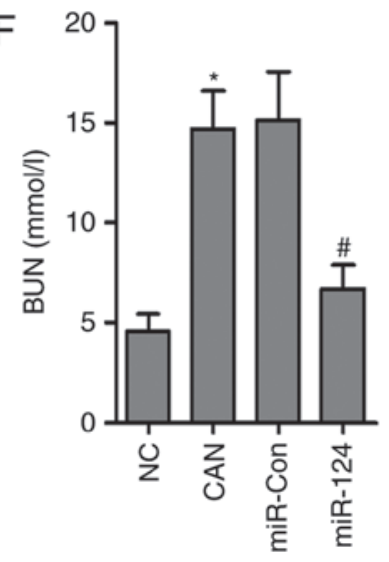

$\mathrm{D}$

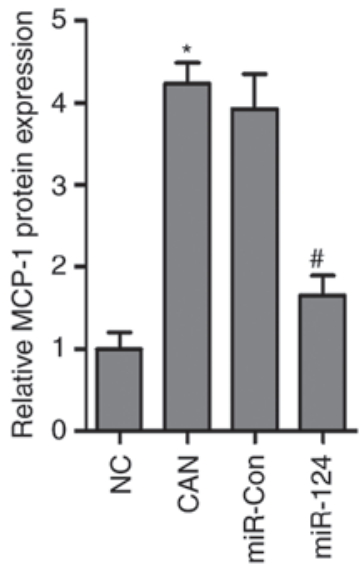

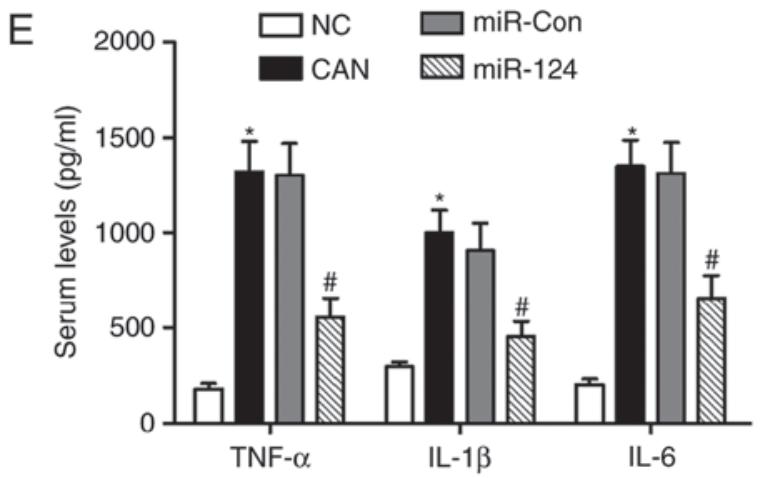

G

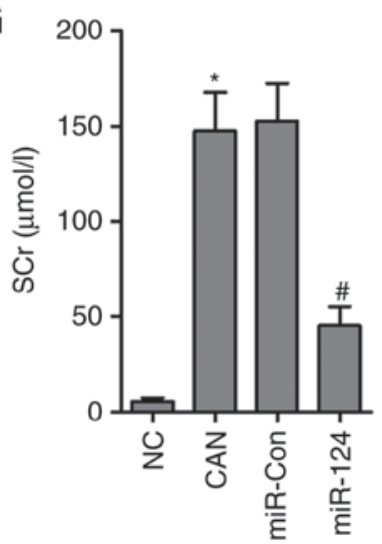

Figure 9. Intravenous injection of miR-124 inhibits the expression of MCP-1, inflammatory cytokines and AKI in septic mice. (A) Levels of miR-124 were analyzed by RT-qPCR in the kidneys of septic mice following adenovirus-delivered miR-124 transfection. mRNA and protein expression were analyzed by (B) RT-qPCR and (C) western blot analyses in the kidneys of septic mice with or without miR-124 treatment. (D) Quantification of blots. (E) Serum levels of inflammatory cytokines, TNF- $\alpha$, IL-1 $\beta$ and IL- 6 were measured using mouse enzyme-linked immunosorbent assay kits in septic mice with or without miR-124 treatment. Levels of (F) BUN and (G) SCr were measured in healthy and septic mice with or without miR-124 treatment. "P<0.05 compared with the NC group; ${ }^{\#} \mathrm{P}<0.05$ compared with the CAN group. miR, microRNA; MCP-1, monocyte chemoattractant protein-1; TNF- $\alpha$, tumor necrosis factor- $\alpha$; IL, interleukin; BUN, blood urea nitrogen; SCr, serum creatinine; RT-qPCR, reverse transcription-quantitative polymerase chain reaction; CAN, Candida albicans; NC, negative control; Con, control.

provided evidence that ITR possessed a nephroprotective effect against candidiasis-induced toxicity via inhibiting the mRNA and protein expression of MCP-1 in the kidney. In candidiasis-exposed mice, significant increases in $\mathrm{BUN}, \mathrm{SCr}$ and mortality rate were observed. By contrast, treatment with ITR resulted in a significant decrease of these parameters in septic mice. On histopathological examination, it was also observed that renal lesions were reduced significantly in septic mice receiving ITR treatment. Unexpectedly, the administration of exogenous miR-124 was able to efficiently restore and ameliorate the damaged kidney in candidemia mice, as ITR, through the suppression of MCP-1 levels. In addition, consistent with a previous study (35), miR-124 directly inhibited the serum levels of TNF- $\alpha$, IL-1 $\beta$ and IL- 6 in septic mice. Therefore, the evidence showed that miR-124 may be a potential therapeutic target for the treatment of inflammatory diseases.

In the present study, it was demonstrated that candidiasis increased the expression of MCP-1 in the kidney and that this effect occurred with the downregulation of miR-124. miR-124 is known to directly target MCP-1, and restoration of the expression of miR-124 by lentiviral infection or formulated miR-124 injection inhibits oral tumor growth in vivo (36). miR-124 is also involved in the amadori-glycated albumin-induced inflammatory effect in cultured rat retinal ganglion cells and the inflammatory phenotype of pulmonary vascular fibroblasts $(37,38)$. The present study confirmed that miR-124 regulated inflammatory signaling via an MCP-1-dependent mechanism in septic mice, associated with renal protection. The results provided confirmation of exogenous miR-124 as a novel avenue for the treatment of AKI.

In our previous study, it was demonstrated that the administration of exogenous miR-204 and miR-211 possessed the ability to efficiently ameliorate candidiasis-induced AKI (22), and is a type of gene therapy that has been well accepted in clinical practice (39). Zhou et al showed that the systemic administration of miR-155 mimics attenuated cardiac dysfunction and improved survival rates in late sepsis (40). The results of a study by Zheng et al showed that the silencing of miR-195 reduced multiple-organ injury and improved survival rates in sepsis (41). All the evidence indicates that exogenous miRs are involved in sepsis-induced inflammatory activity and tissues lesions. In the present study, adenovirus-delivered miR-124 increased by almost 2.5-fold in the kidneys from septic mice, and the overexpression of miR-124 may represent a novel therapeutic approach for sepsis-induced AKI. 
Taken together, previous results and those of the present study provide evidence that MCP-1 is involved in the excessive inflammatory response induced by candidiasis in septic mice. However, ITR had a potent nephroprotective effect on the septic mouse model. In addition, an in vivo experiment demonstrated that adenovirus-delivered miR-124 can be introduced into the kidney to inhibit the expression of MCP-1 and the inflammatory response. These results provide a novel perspective on molecular-targeted therapy for candidiasis-induced AKI.

\section{Competing interests}

The authors declare that they have no competing interests.

\section{References}

1. Leelahavanichkul A, Somparn P, Bootprapan T, Tu H, Tangtanatakul $P$, Nuengjumnong $R$, Worasilchai $N$, Tiranathanagul K, Eiam-ong S, Levine M, et al: High-dose ascorbate with low-dose amphotericin B attenuates severity of disease in a model of the reappearance of candidemia during sepsis in the mouse. Am J Physiol Regul Integr Comp Physiol 309: R223-R234, 2015.

2. Pfaller MA and Diekema DJ: Epidemiology of invasive candidiasis: A persistent public health problem. Clin Microbiol Rev 20: 133-163, 2007.

3. Lionakis MS, Swamydas M, Fischer BG, Plantinga TS, Johnson MD, Jaeger M, Green NM, Masedunskas A, Weigert R, Mikelis C, et al: CX3CR1-dependent renal macrophage survival promotes Candida control and host survival. J Clin Invest 123: 5035-5051, 2013.

4. Spellberg B, Ibrahim AS, Edwards JE Jr and Filler SG: Mice with disseminated candidiasis die of progressive sepsis. J Infect Dis 192: 336-343, 2005.

5. Ngo LY, Kasahara S, Kumasaka DK, Knoblaugh SE, Jhingran A and Hohl TM: Inflammatory monocytes mediate early and organ-specific innate defense during systemic candidiasis. J Infect Dis 209: 109-119, 2014.

6. Navarathna DH, Stein EV, Lessey-Morillon EC, Nayak D, Martin-Manso G and Roberts DD: CD47 promotes protective innate and adaptive immunity in a mouse model of disseminated candidiasis. PLoS One 10: e0128220, 2015.

7. Lionakis MS, Fischer BG, Lim JK, Swamydas M, Wan W, Richard Lee CC, Cohen JI, Scheinberg P, Gao JL and Murphy PM: Chemokine receptor Ccrl drives neutrophil-mediated kidney immunopathology and mortality in invasive candidiasis. PLoS Pathog 8: e1002865, 2012.

8. Castillo L, MacCallum DM, Brown AJ, Gow NA and Odds FC: Differential regulation of kidney and spleen cytokine responses in mice challenged with pathology-standardized doses of Candida albicans mannosylation mutants. Infect Immun 79: 146-152, 2011

9. Imbert F, Jardin M, Fernandez C, Gantier JC, Dromer F, Baron G, Mentre F, Van Beijsterveldt L, Singlas E and Gimenez F: Effect of efflux inhibition on brain uptake of itraconazole in mice infected with Cryptococcus neoformans. Drug Metab Dispos 31: 319-325, 2003.

10. Carrer DP, Samonis G, Droggiti DI, Tsaganos T, Pistiki A and Giamarellos-Bourboulis EJ: Intravenous itraconazole against experimental neutropenic Candida parapsilosis infection: Efficacy after suppression of bacterial translocation. J Infect Chemother 19: 1080-1086, 2013.

11. Sharifzadeh A, Khosravi AR, Shokri H and Tari PS: Synergistic anticandidal activity of menthol in combination with itraconazole and nystatin against clinical Candida glabrata and Candida krusei isolates. Microb Pathog 107: 390-396, 2017.

12. Inoue $H$, Iwasaki $H$, Abe $S$, Yamaguchi $H$ and Ueda $T$ : Modulation of the human interleukin-12p40 response by a triazole antifungal derivative, itraconazole. Scand J Infect Dis 36: 607-609, 2004.

13. Wark PA, Hensley MJ, Saltos N, Boyle MJ, Toneguzzi RC, Epid GD, Simpson JL, McElduff $\mathrm{P}$ and Gibson PG: Anti-inflammatory effect of itraconazole in stable allergic bronchopulmonary aspergillosis: A randomized controlled trial. J Allergy Clin Immunol 111: 952-957, 2003.
14. Oliveira AH, de Oliveira GG, Carnevale Neto F, Portuondo DF Batista-Duharte A and Carlos IZ: Anti-inflammatory activity of Vismia guianensis (Aubl.) Pers. Extracts and antifungal activity against Sporothrix schenckii. J Ethnopharmacol 195: 266-274, 2017.

15. Friccius H, Pohla H, Adibzadeh M, Siegels-Hubenthal P, Schenk A and Pawelec G: The effects of the antifungal azoles itraconazole, fluconazole, ketoconazole and miconazole on cytokine gene expression in human lymphoid cells. Int J Immunopharmacol 14: 791-799, 1992.

16. Ohta K, Ishida Y, Fukui A, Nishi H, Naruse T, Takechi M and Kamata N: Itraconazole inhibits TNF- $\alpha$-induced CXCL10 expression in oral fibroblasts. Oral Dis 21: 106-112, 2015.

17. Naranjo TW, Lopera DE, Diaz-Granados LR, Duque JJ, Restrepo A and Cano LE: Histopathologic and immunologic effects of the itraconazole treatment in a murine model of chronic pulmonary paracoccidioidomycosis. Microbes Infect 12: 1153-1162, 2010.

18. Liu J, Shi K, Chen M, Xu L, Hong J, Hu B, Yang X and Sun R: Elevated miR-155 expression induces immunosuppression via CD39(+) regulatory T-cells in sepsis patient. Int J Infect Dis 40: 135-141, 2015.

19. Pan S, Yang X, Jia Y, Li Y, Chen R, Wang M, Cai D and Zhao R: Intravenous injection of microvesicle-delivery miR-130b alleviates high-fat diet-induced obesity in C57BL/6 mice through translational repression of PPAR- $\gamma$. J Biomed Sci 22: 86, 2015.

20. Ge QM, Huang CM, Zhu XY, Bian F and Pan SM: Differentially expressed miRNAs in sepsis-induced acute kidney injury target oxidative stress and mitochondrial dysfunction pathways. PLoS One 12: e0173292, 2017

21. Monk CE, Hutvagner G and Arthur JS: Regulation of miRNA transcription in macrophages in response to Candida albicans. PLoS One 5: e13669, 2010.

22. Li XY, Zhang K, Jiang ZY and Cai LH: MiR-204/miR-211 downregulation contributes to candidemia-induced kidney injuries via derepression of Hmx1 expression. Life Sci 102: 139-144, 2014.

23. Venturini J, de Camargo MR, Felix MC, Vilani-Moreno FR and de Arruda MS: Influence of tumour condition on the macrophage activity in Candida albicans infection. Scand J Immunol 70: 10-17, 2009.

24. Fan HY, Qi D, Yu C, Zhao F, Liu T, Zhang ZK, Yang MY, Zhang LM, Chen DQ and Du Y: Paeonol protects endotoxin-induced acute kidney injury: potential mechanism of inhibiting TLR4-NF- $\mathrm{B}$ signal pathway. Oncotarget 7: 39497-39510, 2016.

25. Xiao F, Huang Z, Li H, Yu J, Wang C, Chen S, Meng Q, Cheng Y, Gao $\mathrm{X}$ and $\mathrm{Li}$ J: Leucine deprivation increases hepatic insulin sensitivity via GCN2/mTOR/S6K1 and AMPK pathways. Diabetes 60: 746-756, 2011

26. Livak KJ and Schmittgen TD: Analysis of relative gene expression data using real-time quantitative PCR and the 2(-Delta Delta C(T)) method. Methods 25: 402-408, 2001.

27. He J, Chen Y, Lin Y, Zhang W, Cai Y, Chen F, Liao Q, Yin Z, Wang Y, Tao S, et al: Association study of MCP-1 promoter polymorphisms with the susceptibility and progression of sepsis. PLoS One 12: e0176781, 2017.

28. Labbe K, Danialou G, Gvozdic D, Demoule A, Divangahi M, Boyd JH and Petrof BJ: Inhibition of monocyte chemoattractant protein-1 prevents diaphragmatic inflammation and maintains contractile function during endotoxemia. Crit Care 14: R187, 2010.

29. Dorso L, Bigot-Corbel E, Abadie J, Diab M, Gouard S, Bruchertseifer F, Morgenstern A, Maurel C, Chérel M and Davodeau F: Long-term toxicity of 213Bi-labelled BSA in mice. PLoS One 11: e0151330, 2016.

30. Kluger N, Kataja J, Aho H, Ronn AM, Krohn K and Ranki A: Kidney involvement in autoimmune polyendocrinopath y-candidiasis-ectodermal dystrophy in a Finnish cohort. Nephrol Dial Transplant 29: 1750-1757, 2014.

31. Munshi R, Johnson A, Siew ED, Ikizler TA, Ware LB, Wurfel MM, Himmelfarb J and Zager RA: MCP-1 gene activation marks acute kidney injury. J Am Soc Nephro 22: 165-175, 2011.

32. Vianna HR, Soares CM, Silveira KD, Elmiro GS, Mendes PM, de Sousa Tavares M, Teixeira MM, Miranda DM and Simões E Silva AC: Cytokines in chronic kidney disease: Potential link of MCP-1 and dyslipidemia in glomerular diseases. Pediatr Nephrol 28: 463-469, 2013.

33. Yu C, Qi D, Sun JF, Li P and Fan HY: Rhein prevents endotoxin-induced acute kidney injury by inhibiting NF- $\kappa \mathrm{B}$ activities. Sci Rep 5: 11822, 2015. 
34. Chen H, Zhu J, Liu Y, Dong Z, Liu H, Liu Y, Zhou X, Liu F and Chen G: Lipopolysaccharide induces chronic kidney injury and fibrosis through activation of mTOR signaling in macrophages. Am J Nephrol 42: 305-317, 2015.

35. Wang D, Shi L, Xin W, Xu J, Xu J, Li Q, Xu Z, Wang J, Wang G, Yao W, et al: Activation of PPAR $\gamma$ inhibits pro-inflammatory cytokines production by upregulation of miR-124 in vitro and in vivo. Biochem Biophys Res Commun 486: 726-731, 2017.

36. Li X, Fan Q, Li J, Song J and Gu Y: MiR-124 down-regulation is critical for cancer associated fibroblasts-enhanced tumor growth of oral carcinoma. Exp Cell Res 351: 100-108, 2017.

37. Dong N, Xu B, Shi $\mathrm{H}$ and Tang X: Baicalein inhibits amadori-glycated albumin-induced MCP-1 expression in retinal ganglion cells via a microRNA-124-dependent mechanism. Invest Ophthalmol Vis Sci 56: 5844-5853, 2015.
38. Wang D, Zhang H, Li M, Frid MG, Flockton AR, McKeon BA, Yeager ME, Fini MA, Morrell NW, Pullamsetti SS, et al: MicroRNA-124 controls the proliferative, migratory, and inflammatory phenotype of pulmonary vascular fibroblasts. Circ Res 114: 67-78, 2014.

39. Fischer A: Gene therapy: Repair and replace. Nature 510: 226-227, 2014

40. Zhou Y, Song Y, Shaikh Z, Li H, Zhang H, Caudle Y, Zheng S, Yan H, Hu D, Stuart C and Yin D: MicroRNA-155 attenuates late sepsis-induced cardiac dysfunction through JNK and $\beta$-arrestin 2. Oncotarget 8: 47317-47329, 2017.

41. Zheng D, Yu Y, Li M, Wang G, Chen R, Fan GC, Martin C, Xiong $\mathrm{S}$ and Peng T: Inhibition of microRNA 195 prevents apoptosis and multiple-organ injury in mouse models of sepsis. J Infect Dis 213: 1661-1670, 2016. 